\title{
Competitive Oxidation and Protonation of Aqueous Monomethylplatinum(II) \\ Complexes: A Comparison of Oxidants
}

David R. Weinberg, Jay A. Labinger,* and John E. Bercaw*

\section{Supporting Information}

The competitive oxidation and deuterolysis of $\left[\mathrm{Pt}^{\mathrm{II}}\left(\mathrm{CH}_{3}\right) \mathrm{Cl}_{3}\right]^{2-}(\mathbf{1})$ and $\left[\mathrm{Pt}^{\mathrm{II}}\left(\mathrm{CH}_{3}\right)_{2} \mathrm{Cl}_{2}\right]^{2-}(\mathbf{4})$ at $22{ }^{\circ} \mathrm{C}$ in $\mathrm{D}_{2} \mathrm{O}$ solutions.

Table 1. Dependence of $k_{O_{x}} / k_{D^{+}}$and $k_{O x} / k_{H^{+}}$on $\mathrm{Na}_{2}\left[\mathrm{Pt}^{\mathrm{IV}} \mathrm{Cl}_{6}\right]\left(\left[\mathrm{D}_{2} \mathrm{SO}_{4}\right]=0.35 \mathrm{M},\left[\mathrm{Cl}^{-}\right]=3.0 \mathrm{M}\right)$

\begin{tabular}{|c|c|c|c|c|}
\hline $\mathrm{Na}_{2}\left[\mathrm{Pt}^{\mathrm{IV}} \mathrm{Cl}_{6}\right]$ & $k_{o x} / k_{D+}$ & $k_{o x} / k_{H+}$ & $k_{o x} / k_{D+}$ & $k_{o x} / k_{H^{+}}$ \\
\hline$(\mathrm{M})$ & {$\left[\mathrm{Pt}^{\mathrm{II}}\left(\mathrm{CH}_{3}\right) \mathrm{Cl}_{3}\right]^{2-}$} & {$\left[\mathrm{Pt}^{\mathrm{II}}\left(\mathrm{CH}_{3}\right) \mathrm{Cl}_{3}\right]^{2-}$} & {$\left[\mathrm{Pt}^{\mathrm{II}}\left(\mathrm{CH}_{3}\right)_{2} \mathrm{Cl}_{2}\right]^{2-}$} & {$\left[\mathrm{Pt}^{\mathrm{II}}\left(\mathrm{CH}_{3}\right)_{2} \mathrm{Cl}_{2}\right]^{2-}$} \\
\hline 0.12 & 3.2 & 0.35 & 4.1 & 0.45 \\
\hline 0.12 & 3.4 & 0.37 & 4.5 & 0.50 \\
\hline 0.12 & 4.1 & 0.46 & 5.8 & 0.64 \\
\hline 0.12 & 3.3 & 0.36 & 5.8 & 0.64 \\
\hline 0.25 & 3.6 & 0.40 & 4.0 & 0.44 \\
\hline 0.37 & 3.2 & 0.36 & 3.2 & 0.35 \\
\hline 0.37 & 2.5 & 0.27 & 3.3 & 0.37 \\
\hline 0.50 & 2.6 & 0.29 & 2.8 & 0.31 \\
\hline 0.50 & 4.1 & 0.46 & 3.5 & 0.39 \\
\hline \hline $\begin{array}{c}\text { average* } \\
\text { standard } \\
\text { deviation* }\end{array}$ & 3.3 & 0.37 & 4.1 & 0.46 \\
\hline
\end{tabular}

*These numbers differ from those reported in the text: the latter are based only on the data for $\left[\mathrm{Na}_{2} \mathrm{Pt}^{\mathrm{IV}} \mathrm{Cl}_{6}\right]=0.12 \mathrm{M}$, for the purpose of using the same conditions to compare low and high $[\mathrm{Cl}]^{-}$. 
Table 2. Dependence of $k_{O_{x}} / k_{D^{+}}$and $k_{O_{x}} / k_{H^{+}}$on $\left[\mathrm{Cl}^{-}\left(\mathrm{Na}_{2}\left[\mathrm{Pt}^{\mathrm{IV}} \mathrm{Cl}_{6}\right]=0.12 M,\left[\mathrm{D}_{2} \mathrm{SO}_{4}\right]=0.35 M\right)\right.$

\begin{tabular}{|c|c|c|c|c|}
\hline$\left[\mathrm{Cl}^{-}\right]$ & $k_{o x} / k_{D^{+}}$ & $k_{o x} / k_{H^{+}}$ & $k_{o x} / k_{D^{+}}$ & $k_{o x} / k_{H^{+}}$ \\
\hline$(\mathrm{M})$ & {$\left[\mathrm{Pt}^{\mathrm{II}}\left(\mathrm{CH}_{3}\right) \mathrm{Cl}_{3}\right]^{2-}$} & {$\left[\mathrm{Pt}^{\mathrm{II}}\left(\mathrm{CH}_{3}\right) \mathrm{Cl}_{3}\right]^{2-}$} & {$\left[\mathrm{Pt}^{\mathrm{II}}\left(\mathrm{CH}_{3}\right)_{2} \mathrm{Cl}_{2}\right]^{2-}$} & {$\left[\mathrm{Pt}^{\mathrm{II}}\left(\mathrm{CH}_{3}\right)_{2} \mathrm{Cl}_{2}\right]^{2-}$} \\
\hline \hline 0.72 & 8.099 & 0.900 & 8.55 & 0.95 \\
\hline 0.72 & 10.877 & 1.209 & 9.21 & 1.02 \\
\hline 0.72 & 6.871 & 0.763 & 12.54 & 1.394 \\
\hline 0.72 & 7.723 & 0.858 & 16.44 & 1.827 \\
\hline 0.72 & 8.625 & 0.958 & 14.23 & 1.582 \\
\hline 1.5 & 5.677 & 0.631 & 6.70 & 0.74 \\
\hline 2.2 & 4.521 & 0.502 & 6.06 & 0.67 \\
\hline 3.0 & 3.165 & 0.352 & 4.09 & 0.45 \\
\hline 3.0 & 3.352 & 0.372 & 4.49 & 0.499 \\
\hline 3.0 & 4.125 & 0.458 & 5.78 & 0.642 \\
\hline 3.0 & 3.282 & 0.365 & 5.77 & 0.641 \\
\hline
\end{tabular}

Table 3. Dependence of $k_{O x} / k_{D^{+}}$and $k_{O x} / k_{H^{+}}$on $[\mathrm{Cl}]^{-}\left(\left[\mathrm{Cu}^{\mathrm{II}} \mathrm{Cl}_{2}\right]=0.25 \mathrm{M},\left[\mathrm{D}_{2} \mathrm{SO}_{4}\right]=0.35 \mathrm{M}\right)$

\begin{tabular}{|c|c|c|c|c|}
\hline$\left[\mathrm{Cl}^{-}\right]$ & $k_{o x} / k_{D+}$ & $k_{o x} / k_{H^{+}}$ & $k_{o x} / k_{D^{+}}$ & $k_{o x} / k_{H^{+}}$ \\
\hline$(\mathrm{M})$ & {$\left[\mathrm{Pt}^{\mathrm{II}}\left(\mathrm{CH}_{3}\right) \mathrm{Cl}_{3}\right]^{2-}$} & {$\left[\mathrm{Pt}^{\mathrm{II}}\left(\mathrm{CH}_{3}\right) \mathrm{Cl}_{3}\right]^{2-}$} & {$\left[\mathrm{Pt}^{\mathrm{II}}\left(\mathrm{CH}_{3}\right)_{2} \mathrm{Cl}_{2}\right]^{2-}$} & {$\left[\mathrm{Pt}^{\mathrm{II}}\left(\mathrm{CH}_{3}\right)_{2} \mathrm{Cl}_{2}\right]^{2-}$} \\
\hline 0.496 & 0.00 & 0.00 & 44.25 & 4.92 \\
\hline 3.0 & 0.776 & 0.086 & 48.41 & 5.38 \\
\hline
\end{tabular}

